\title{
Underconfidence in sensory discrimination: The interaction between experimental setting and response strategies
}

\author{
HENRIK OLSSON and ANDERS WINMAN \\ Uppsala University, Uppsala, Sweden
}

\begin{abstract}
In a recent issue of this journal, Baranski and Petrusic (1994) presented empirical data revealing overconfidence in sensory discrimination. In this paper, we propose an explanation of Baranski and Petrusic's results, based on an idiosyncrasy in the experimental setting that misleads subjects who are using an unwarranted symmetry assumption. Experiment 1 showed that when this hypothesis is controlled for, a large underconfidence bias is obtained with Baranski and Petrusic's procedure. The results of Experiment 2 confirmed that overconfidence is difficult to obtain in subject-controlled sensory discrimination tasks, even for a very low proportion of correct responses. The different results obtained in sensory and cognitive tasks suggest that one should not uncritically draw parallels between confidence in sensory and cognitive judgments.
\end{abstract}

In a recent article in this journal, Baranski and Petrusic (1994; henceforth, B\&P) proposed that there is no real difference in realism of confidence judgments between the sensory and cognitive domains. They argued that there is underconfidence in the sensory domain only when discriminations are relatively easy, and they presented empirical data in which overconfidence seemed to replace underconfidence for results less than $80 \%$ correct. This observation contrasts with the suggestion made by Björkman, Juslin, and Winman (1993) that underconfidence in sensory discrimination is a robust phenomenon. Björkman et al. (1993) presented data revealing a pervasive underconfidence bias for visual judgment of length and heaviness, with a proportion of correct responses ranging from $67 \%$ to $78 \%$.

Other studies have indicated that it is difficult to obtain overconfidence in sensory discrimination. On the basis of three experiments with a proportion of correct responses ranging from $67 \%$ to $79 \%$, Keren (1988) concluded that "no overconfidence" was "observed in these perceptual tasks" (p. 115). Dawes's (1980) results were not quite conclusive, but they indicated that "the finding of overconfidence using intellectual content can be reversed with some subjects making some perceptual judgments" (p. 344). The proportion of correct responses obtained by Dawes ranged from $71 \%$ to $79 \%$. Some of the tasks in the studies by Dawes and Keren were not purely discriminative in nature (e.g., one of the tasks in Dawes, 1980, was to remember the eye

This research was supported by the Swedish Council for Research in the Humanities and the Social Sciences. Our thanks to Mats Björkman, Peter Juslin, Emily Holmes, Lester E. Krueger, William M. Petrusic, and an anonymous reviewer for their valuable comments on an earlier draft of this paper. Correspondence concerning this paper should be addressed to H. Olsson, Department of Psychology, Uppsala University, Box 1854, S-751 48 Uppsala, Sweden (e-mail: henrik.olsson@psyk.uu.se). color of university professors), whereas Björkman et al. (1993) used stimuli of varying magnitude on the same sensory continua and pairwise comparisons with the method of constant stimuli. Furthermore, in some studies, subjects are given the opportunity to inspect stimuli for as long as they wish (e.g., Björkman et al., 1993), whereas in others, the experimenter controls stimulus duration (e.g., Experiment 3 in B\&P; see Vickers, Smith, Burt, \& Brown, 1985 , for a discussion of the differences between subjectcontrolled and experimenter-controlled situations). When we talk of subject- and experimenter-controlled situations, we are referring to the control of the duration of the stimuli: the term subject-controlled refers to studies in which subjects are given the opportunity to inspect stimuli as long as they wish; in experimenter-controlled studies, the experimenter controls stimulus duration. These differences can be crucial in determining what cognitive and sensory processes underlie the probability judgments. It is our belief that if subjects are given "fair" circumstances and time to inspect stimuli, they will in most cases be underconfident for pairwise comparisons on a single continuum. Therefore, in the present study, all tasks were subject controlled; subjects could inspect stimuli for as long as they wished.

In the cognitive domain, the most frequently reported result is overconfidence (for reviews, see Keren, 1991; Lichtenstein, Fischhoff, \& Phillips, 1982; O'Connor, 1989; Wallsten \& Budescu, 1983; Yates, 1990). However, under certain conditions, close to zero over-/underconfidence $(\mathrm{O} / \mathrm{U})$ has been reported (Gigerenzer, Hoffrage, \& Kleinbölting, 1991; Juslin, 1993a, 1993b, 1994, 1995; Juslin, Winman, \& Persson, 1995; Winman, in press).

The claims in B\&P are important to scrutinize both from a general methodological perspective and in the light of the current debate on the status of the proposed underconfidence phenomenon in sensory discrimination (see, e.g., Björkman et al., 1993, 1995; Ferrell, 1994, 1995; Juslin \& 
Winman, 1995). We believe that B\&P overlooked a possible explanation of their results. The explanation put forward here is based on the hypotheses that (1) subjects are using a strategy based on a symmetry assumption, and (2) a possible source of error in B\&P's experimental setting systematically misled the subjects. The experiments in this paper show that when these considerations are accounted for, a large underconfidence bias is in fact obtained with the procedure reported in B\&P. This paper focuses on conditions when subjects are given neither time limits nor feedback. Our interest is specifically directed at Experiment 2 in B\&P, since that study showed particularly intriguing results.

\section{One Possible Explanation of the Results in Baranski and Petrusic's (1994) Experiment 2}

In B\&P's Experiment 2, two short vertical lines were presented at varying distances from a central line that was slightly longer (see Figure 1). The subjects were to judge which of the two lines, the left or the right, was nearer to/farther from the central line and indicate their confidence in their decisions.

$B \& P$ found a high degree of overconfidence toward the most difficult stimulus combination, and they found that discriminative accuracy depended on which line in the pair was located nearer to the midline. The subjects were much less accurate when the line on the left was the element in the pair that was nearer to the central line-as in the case of the pair $(296,300)$, for example, where the two numbers indicate how many pixels the lines are from the central marker. Calibration curves (i.e., proportion of correct responses plotted against confidence) showed that for the stimulus pair $(296,300)$, proportion correct decreased with confidence; subjects who were $100 \%$ certain were much less likely to be correct (approximately $22.5 \%$ correct) than subjects who reported guessing (approximately $38 \%$ correct) (see Figure 7 in $\mathrm{B} \& \mathrm{P}$ ). This positional-order effect was so pronounced for the pair $(296,300)$ that it led to a proportion of correct responses significantly lower than chance: .255 and an $\mathrm{O} / \mathrm{U}$ of .524 . $(\mathrm{O} / \mathrm{U}$ is defined as $\bar{x}-\bar{c}$, where $\bar{c}$ is the proportion of correct responses and $\bar{x}$ is the mean confidence. A positive $\bar{x}-\bar{c}$ indicates overconfidence; a negative $\bar{x}-\bar{c}$, underconfidence.)

For the pair $(300,296)$, where the left stimulus is farthest from the central line, the data look entirely different. The proportion of correct responses was very high, .871; it increased with confidence; and underconfidence was observed, $\mathrm{O} / \mathrm{U}=-.071$. A similar positional-order effect was found for another stimulus pair $(70,69 ; 69,70)$. $B \& P$ gave no further explanation of this effect aside from a general reference to perceptual illusions and complex response biases (see, e.g., pp. 421 and 424). In other words, the subjects in B\&P's Experiment 2 thought more often that the left line was located farther from the central line than the right line. It is our belief that this positional-order effect is in fact not merely an intriguing curiosity, but the very key to an understanding of B\&P's results.

In recreating the experimental situation in $\mathrm{B} \& \mathrm{P}$ and running some pretests on ourselves and colleagues, two things became evident. First, we realized that the display of visual stimuli on a computer screen is a delicate business. It was no straightforward task to place a stimulus in a certain location by using a simple programmed code instruction. In testing the setup on different computers, very large discrepancies in the location of the screen center relative to the monitor edges were found. Even modern screens suffer from quite large nonlinearities as well as temporary fluctuations. In order to position stimuli where they ought to be, careful physical measurements are necessary. For example, on one new monitor, we estimated from the resolution capability that a line would be drawn $100 \mathrm{~mm}$ to the left, but when we measured the actual distance it turned out to be $103 \mathrm{~mm}$ to the left. Second, it became evident that people often use a particular strategy when confronted with the task. In the experimental setting some stimuli are close to the edges of the computer screen. Instead of judging the length to the central line, subjects reported estimating the two distances to the sides of the monitor. That is, they judged the distances $c$ and $d$ instead of $a$ and $b$ in Figure 1 .

Why would it be more reasonable for subjects to estimate the distances to the side of the monitor rather than to the central line? An example with the distances in the pair $(296,300)$ will illustrate this: The ratio $r(a / b)$ (or $b / a$ if $b>a$ ) is used to denote the level of difficulty for a specific stimulus combination (note that it is the ratios that are important, rather than the differences, $a-b$ and $c-d$, following the relative principle in Weber's law). A low value indicates a difficult stimulus combination. From the information in B\&P's (1994) Method section we estimated their screen width to be $230 \mathrm{~mm}$ and then calculated the values of $a, b, c$, and $d$, on the basis of the presumption that $a+c=b+d=115 \mathrm{~mm}$. With the pair $(296,300)$, $a=94.6 \mathrm{~mm}$ and $b=95.8 \mathrm{~mm}$. This makes $r=1.01$. The distances $c=20.4$ and $d=19.2$, on the other hand, result in a much higher and easier $r$ of 1.06. Subjects might have been using this "shortcut" as a more readily discriminable possibility. (This strategy will be referred to as the edge strategy in the following.) Thus, it is more efficient to use $c$ and $d$ to estimate the length to the central line when the

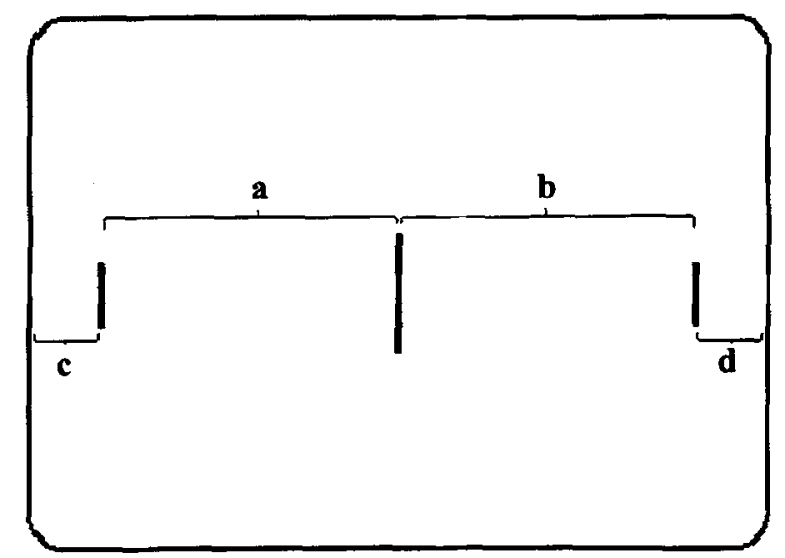

Figure 1. The task in Baranski and Petrusic's (1994) Experiment 2 . 
lines are closer to the monitor edge than to the central line. The point here is that subjects make the reasonable assumption that the central line is located exactly in the center of the screen. Note that the terminology used here, strategy or inference, is not meant to imply that the subjects have complete conscious control of the edge strategy. Even if subjects are aware of the fact that the screen is off center relative to the monitor edges, it can be difficult for them to completely ignore the distances to the monitor edges. In our pretests, subjects reported that it was difficult not to use the strategy even if they were told that the screen was off center relative to the monitor edges. It seems that the distances $c$ and $d$ are much too salient to ignore, at least for stimuli presented close to the monitor edges. If the use of the edges is to be eliminated totally, we need an experimental setting where the monitor edges are not visible at all (this manipulation was undertaken in Experiment 2 below).

What would happen if the screen happened to be slightly off center relative to the monitor edges? Subjects would be misled by the experimental setting, with a decrease in the proportion of correct responses. If the off-centering occurred in the left direction, we would expect a positionalorder effect like the one observed by B\&P. The left line would appear to be farther away from the central line because it would appear to be closer to the left monitor edge. If the off-centering was in the right direction, we would expect a mirror image of the positional-order effect found by $\mathrm{B} \& \mathrm{P}$; the right line would look farthest from the central line. It is difficult to give an exact estimate of how large an off-centering of the screen would have to be in order to yield results comparable to those of B\&P. Presumably, it would have to be larger than $1.2 \mathrm{~mm}$, because this value implies that the distances $c$ and $d$ are the same for the pair $(296,300)$. This degree of off-centering would, if subjects relied solely on the edge strategy, lead to a proportion of correct responses of approximately .5 (as noted above, $\mathrm{B} \& \mathrm{P}$ reported .255 for this pair).

In this way, the positional-order effect found by $B \& P$ could be explained by a slightly off-center screen in relation to the monitor edges. We hypothesize that if the screen is experimentally off-centered to the left, the same pattern as in B\&P will be observed. When subjects try to judge the distances $c$ and $d$ (see Figure 1) when the left line is nearer to the central line, they will make more incorrect responses than correct responses (e.g., for Pair 6, [296, $300]$ ), because $c>d$ in the centered version depicted in Figure 1 will have changed to $c<d$. If the left line is farther from the central line, there will be more correct responses than incorrect responses (e.g., the other presentation order for Pair 6, [300, 296]). This will also (given a degree of off-centering larger than the physical stimulus difference) lead to higher confidence, since the distances $c$ and $d$ will influence the confidence assessments, with many responses occurring in the $100 \%$ category. The lower magnitude of the positional-order effect found in the $(69,70)$ pair can be explained by the fact that the subjects use the edge strategy to a lesser extent when the lines are closer to the central line.

\section{The Present Study}

Three questions are of primary interest in the present study: (1) Can we replicate B\&P's finding of overconfidence when subjects are presented with perfectly centered stimuli combinations? (2) Can underconfidence be obtained even in a very difficult task? (3) Can a positional-order effect be obtained if we preclude the subjects from relying on the edge strategy? In Experiment 1, we were mainly concerned with the first question, and in Experiment 2, with the other two questions.

Experiment 1 was essentially a replication of B\&P's Experiment 2, but to test the explanation given above, we divided the subjects into three groups: One had the stimulus combinations exactly centered, another had the stimulus combinations off center to the left, and a third had the stimulus combinations off center to the right. In Experiment 2, a box with a viewing hole was placed around the monitor to make it impossible to use the monitor edges as referents. If there exists a positional-order effect that is not produced by the interaction between the edge strategy and the positioning of the stimuli combinations relative to the monitor edges, the effect should become evident under the more controlled conditions in Experiment 2 . B\&P suggested that the hard-easy effect (i.e., overconfidence for tasks with a proportion of correct responses below $80 \%$ and underconfidence for tasks with a proportion of correct responses above $80 \%$ ) that is commonly observed in the cognitive domain applies to sensory tasks as well. The task in Experiment 1 was quite easy, so we made Experiment 2 more difficult in order to make the evaluation of B\&P's hard-easy claim more conclusive.

\section{EXPERIMENT 1}

Experiment 1 was conducted to test whether the explanation above would be sufficient to explain B\&P's result and to see whether it would be possible to replicate the finding of overconfidence for hit rates below $80 \%$. We recreated the experimental conditions in B\&P's Experiment 2 and, in addition, made it possible to off-center the stimulus combinations to the left and to the right, relative to the monitor edges. We hypothesized as follows:

1 . The use of a screen that is off center to a certain degree relative to the monitor edges will lead to higher confidence ratings than will a screen that is centered (control condition). This is because subjects' assessments are probably influenced by the higher ratios that are obtained when subjects compare the distances $c$ and $d$ in Figure 1 .

2 . When the screen is not centered, a lower proportion of correct responses is expected than in the control condition, since the edge strategy will not be reliable under these circumstances.

3. It follows from (1) and (2) that less underconfidence will be observed in an off-center condition, or possibly overconfidence in the hardest stimulus conditions.

4. Off-centering the screen to the left will give the same positional-order effect as in B\&P. When the screen is off center to the right, the opposite pattern is expected. In the 
control condition, no particular positional-order effect is expected.

5. In a control condition, which is predicted to have higher proportion correct and lower confidence than the off-center conditions, underconfidence is expected for all three levels of difficulty.

Apart from testing these five hypotheses, we examined another phenomenon reported in the calibration literature: the "confidence-frequency effect" (Gigerenzer et al., 1991). This effect consists of the finding that in cognitive tasks subjects can be overconfident when they make single confidence judgments, yet they can still be realistic when asked to estimate their total percentage correct after the experiment. Whether this effect occurs also for sensory tasks is unknown. For this purpose, subjects were asked to rate their total percentage correct after taking part in Experiment 1.

\section{Method}

Subjects. Forty-two undergraduate students of Uppsala University ( 18 males and 24 females; mean age, 23.3 years) participated in the study. The subjects did not receive monetary reward, but they were given credit for a course requirement. All had normal or correctedto-normal vision. They were not informed of the purpose of the experiment, which lasted about $90 \mathrm{~min}$. A between-subjects design was used, with subjects randomly assigned to the three experimental conditions ( 14 subjects in each group): stimulus combinations centered $(C)$, stimulus combinations set left (L), and stimulus combinations set right $(\mathrm{R})$.

Apparatus. The stimuli were presented on a MAG DX 17 F, 17 in., flat-screen monitor with a resolution capability of 1,280 pixels wide $\times 1,024$ pixels high. An IBM-compatible personal computer was used to randomize and generate the stimuli and record the responses. The subjects responded with a mouse. Confidence responses were given on the keyboard. A small desk lamp positioned behind the monitor provided sufficient light for responding without interfering with the visual display.

Stimuli. The stimulus display consisted of two $5-\mathrm{mm}$ vertical lines presented on the left and right of a 10 -mm vertical central fixation line. This line served the purpose of allowing a reference from which to perform the comparative judgment and to divide the screen into left and right sides. The stimulus combination was positioned exactly in the middle (C), $3 \mathrm{~mm}$ to the left of the center (L), and $3 \mathrm{~mm}$ to the right of the center $(R)$ in each of the experimental conditions, respectively. The $3-\mathrm{mm}$ deviation from the center of the screen was arbitrarily chosen so as not to be obvious, but to still stand a chance of giving an effect. All lines were $1 \mathrm{~mm}$ wide; they were amber in color against a black background.

The notation $(x, y)$ is used to denote a stimulus pair $x$ pixels $(2.23$ pixels $=1 \mathrm{~mm}$ ) to the left of the referent and $y$ pixels to the right. All stimuli were scaled up by a factor of 1.4 from the distances used by $B \& P$, preserving their ratios. This was done to proportionally preserve the distances from the stimuli to the outer screen edge of the monitor which are of considerable interest in the present study. It was deduced from the screen's horizontal resolution capabilities (720 pixels) and the width of a single pixel $(3.13$ pixels $=1 \mathrm{~mm}$ ) that B\&P used a monitor with an approximate width of $230 \mathrm{~mm}$. (Monitor size is not explicitly reported in their article.) All stimuli were carefully measured directly on the monitor screen in order to position them in their exact location. The two presentation orders of the six pairs constructed by $\mathrm{B} \& \mathrm{P}$ for their second experiment were used in the present experiment. These were $(30,28 ; 28,30) ;(50,48$; $48,50) ;(70,69 ; 69,70) ;(260,243 ; 243,260) ;(280,269 ; 269,280)$; and $(300,296 ; 296,300)$. The difficulty of these stimulus pairs is expressed in terms of the ratio $r$ of the longer to the shorter element: $1.07,1.04,1.01,1.07,1.04$, and 1.01 for Pairs $1-6$, respectively. The ratio 1.07 represents the easiest comparisons; 1.04 represents the intermediate level; and 1.01 represents the hardest comparisons. At a viewing distance of approximately $80 \mathrm{~cm}$, the distances of the nearest and farthest stimulus pairs subtended visual angles of approximately $2^{\circ}$ and $19^{\circ}$, respectively, approximately the same as in B\&P's study.

Procedure. Each trial began with the presentation of a label (NEARER or FARTHER) centered near the top of the screen, followed $1.5 \mathrm{sec}$ later by the stimulus pair. Both the instruction label and the pair remained on the screen until the subject responded. The task required subjects to judge which of the two small vertical lines was, depending on the current label, either nearer or farther from the central line. They responded by pressing either the left or the right button on the mouse, after which the screen was cleared and a visual prompt appeared (CONFIDENCE $\rightarrow$ ). Subjects then typed a confidence rating from 0 to 100 , using the numeric keypad on the keyboard. Prior to the experiment, the subjects were given detailed instructions on how to use this confidence scale. A rating of $100 \%$ indicated $a b$ solute certainty that a correct response had been made, and a rating of $50 \%$ indicated that $a$ response was a guess. Ratings between $51 \%$ and $99 \%$ were to represent increasing confidence (expressed as a probability or likelihood) that a correct response had been made. Confidence ratings between $0 \%$ and $49 \%$ were to be used only on trials in which subjects felt they had made an error, with $0 \%$ denoting absolute certainty of an error response. There was no time limit for registering the confidence report. Subjects did not receive feedback on the accuracy of the judgments. A 2-sec interval with the screen blank separated the trials. Subjects began with six practice trials, followed by two blocks of 192 randomized trials separated by a short break. This made up a total of 384 trials: 6 pairs $\times 2$ response modes (NEARER or FARTHER) $\times 2$ presentation orders $\times 16$ replications $=384$.

\section{Results}

A total of $133(0.8 \%)$ of 16,128 responses resulted in a confidence below $50 \%$ and were excluded from the analysis. The data were collapsed over FARTHER and NEARER trials, since no significant differences were found for either proportion correct or confidence. The confidence categories were obtained in the same way as in B\&P: $50 \%-59 \%$, $60 \%-69 \%, 70 \%-79 \%, 80 \%-89 \%, 90 \%-99 \%$, and $100 \%$. The main results of Experiment $l$ are reported in Table 1. It can be seen that in the $C$ condition (with the stimulus combinations exactly centered) there is a large underconfidence that is more than three times as large as in both the $\mathrm{L}$ and $\mathrm{R}$ conditions. Planned comparisons tests show that the $\mathrm{C}$ group has a significantly higher proportion of correct responses than do the $\mathrm{L}$ and $\mathrm{R}$ groups $[t(39)=3.28$, $p<.005]$. At the same time, the $\mathrm{C}$ group has significantly lower mean confidence ratings than do the $\mathrm{L}$ and $\mathrm{R}$ groups $[t(39)=2.05, p<.05]$. This also leads to the C group's having a significantly lower $\mathrm{O} / \mathrm{U}$ score than those of the other two groups $[t(39)=3.68, p<.001]$.

The data can also be presented in calibration curves (i.e., the proportion of correct responses in each of the six confidence categories), as in Figure 2a. ${ }^{1}$ Here, underconfidence is represented by points above the main diagonal, and overconfidence is represented by points below the diagonal. The difference between the groups is evident in the calibration curves of Figure $2 a$, where the $C$ group lies above the others throughout all response categories. As is shown in Figure 2b, the response distribution is much different in the $\mathrm{C}$ group than in the experimental groups. 
Table 1

Experiment 1: Mean Confidence $(\bar{x})$, Proportion Correct $(\bar{c})$, and Over-/Underconfidence $(O / U$, i.e., $\vec{x}-\bar{c})$ from Experiment 2 in B\&P (1994) and in the $C, L$, and $R$ Conditions for Three Levels of Judgment Difficulty: $\mathrm{L} 1(r=1.07), \mathrm{L2}(r=1.04)$, and L3 $(r=1.01)$

\begin{tabular}{lcccc}
\hline Level & $\bar{x}$ & $\bar{c}$ & $\mathrm{O} / \mathrm{U}(\bar{x}-\bar{c})$ \\
\hline & & $\mathrm{B} \& \mathrm{P}$ & \\
L1 & .810 & & .887 & -.077 \\
L2 & .779 & & .766 & .013 \\
L3 & .758 & & .589 & .169 \\
All & .782 & & .747 & .035 \\
& & $\mathrm{C}$ & & \\
L1 & .710 & & .953 & -.243 \\
L2 & .644 & & .900 & -.256 \\
L3 & .593 & & .686 & -.093 \\
All & .649 & & .846 & -.197 \\
& & $\mathrm{~L}$ & & \\
L1 & .770 & & .904 & -.134 \\
L2 & .716 & & .824 & -.108 \\
L3 & .676 & & .611 & .065 \\
All & .720 & & .780 & -.060 \\
& & $\mathrm{R}$ & & \\
L1 & .730 & & .858 & -.128 \\
L2 & .695 & & .785 & .090 \\
L3 & .678 & & .629 & -.056 \\
All & .701 & & .757 & \\
\hline
\end{tabular}

Note-B\&P, Baranski and Petrusic (1994); C, stimulus combinations in middle; $\mathrm{L}$, stimulus combination $3 \mathrm{~mm}$ to left of center; $\mathrm{R}$, stimulus combination $3 \mathrm{~mm}$ to right of center.

There are markedly more responses in the guessing category and fewer in the " $100 \%$ " category in the former.

Difficulty levels. Table 1 also shows the results of the three different difficulty levels, compared with the data from B\&P. In the C group there is underconfidence for all levels of difficulty, whereas the $L$ and $R$ groups reveal overconfidence for the hardest items. This overconfidence is not, however, as large as the overconfidence found by $B \& P$. In the hard subset, there is a difference in response distributions between the $\mathrm{C}$ group and the other groups. In the $\mathrm{C}$ group, there are hardly any (only 12 out of 1,783 , or $0.67 \%$ ) responses in the $100 \%$ category, whereas this response is 13 times $(310 / 3,555=8.7 \%)$ more common in the other two groups.

Stimulus pair subsets. In order to compare the results of the present study with the results in B\&P showing a positional-order effect, a separate analysis of Stimulus Pairs 3 and 6 was performed. Results of this analysis are found in Table 2. In the $\mathrm{C}$ condition, there is underconfidence in all subsets, regardless of whether the farthest stimulus is presented to the left or to the right on the screen. Pair 6 shows a tendency for a positional-order effect with a higher proportion of correct responses when the farthest stimulus is presented to the left. This pattern is reversed for Pair 3, where there is a higher proportion of correct responses when the farthest stimulus is presented to the right. For the $\mathrm{L}$ and $\mathrm{R}$ groups, the pattern is entirely different. When the left stimulus is nearest to the central line, there is overconfidence in the L group and underconfi- dence in the $R$ group, and when the right stimulus is nearest to the central line, there is overconfidence in the $R$ group and underconfidence in the $\mathrm{L}$ group. Most extreme is the overconfidence in the $(300,296)$ pair for the $\mathrm{R}$ condition and in the $(296,300)$ pair for the $L$ condition. These conditions are associated with calibration curves in which the proportion of correct responses decreases with increasing confidence. Figure 3 shows the calibration curves of the stimulus Pair 6 when (a) the right line is farthest from the central line and (b) the left line is farthest from the central line. (B\&P's data are estimated from their Figure 7). The figure clearly illustrates that the pattern for the L condition is almost identical to that from $B \& P$. The curves of the $\mathrm{R}$ condition, on the other hand, look like mirror images of the $L$ condition and B\&P's data.

Verbal reports. After the experiment, subjects were asked if they had applied any strategy in solving the task. If they answered "no" to this question, they were explicitly asked whether they had used the monitor edges in the
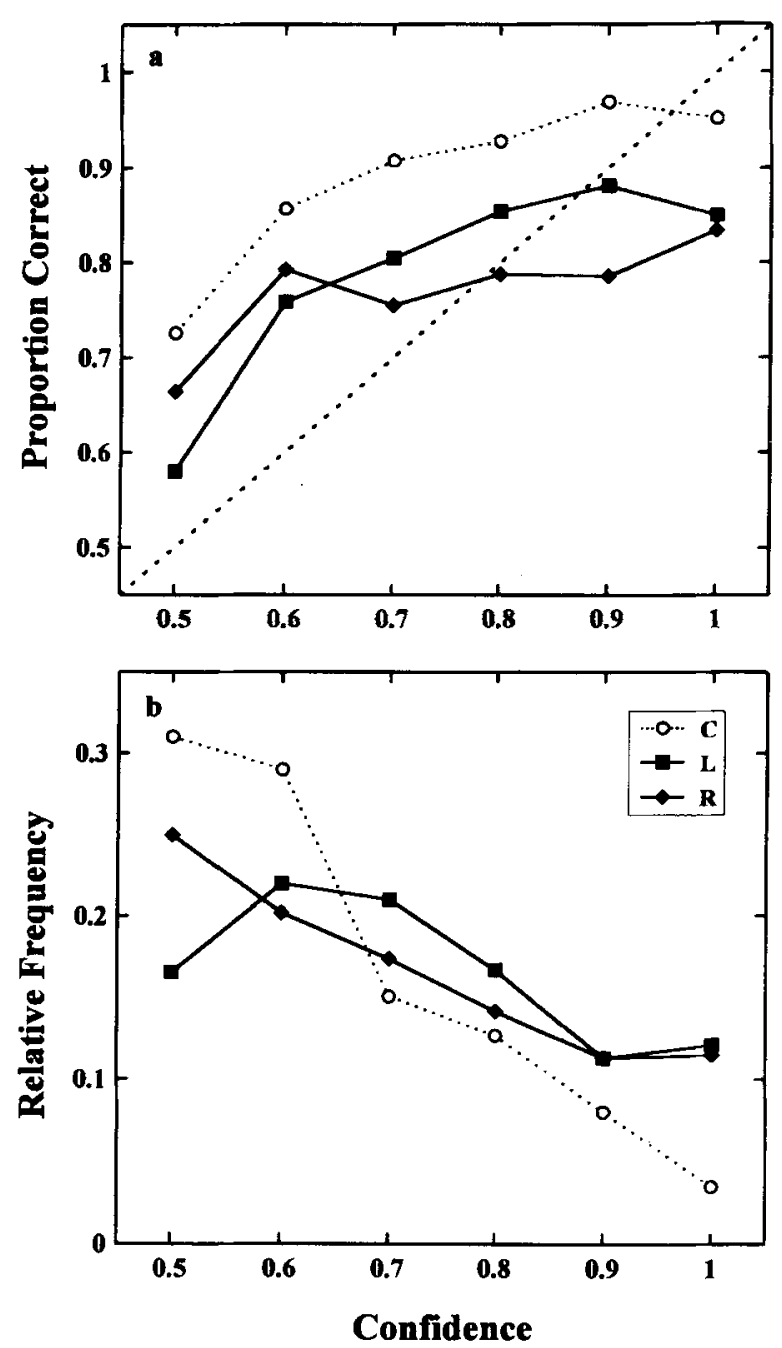

Figure 2. Experiment 1: (a) calibration curves and (b) response frequencies for the $C, L$, and $R$ conditions. 
Table 2

Experiment 1: Mean Confidence $(\bar{x})$, Proportion Correct $(\bar{c})$, and Over-/Underconfidence $(O / U$, i.e., $\bar{x}-\bar{c})$ From Experiment 2 in B\&P (1994) and in the $C$, L, and $R$ Conditions for the Two Pairs in the Most Difficult Level

\begin{tabular}{llccr}
\hline Pair & Order, Pixels & $\bar{x}$ & $\bar{c}$ & $\mathrm{O} / \mathrm{U}(\bar{x}-\bar{c})$ \\
\hline \multirow{4}{*}{3} & 70,69 & B\&P & & \\
& 69,70 & .784 & .673 & .111 \\
6 & 300,296 & .780 & .551 & .229 \\
& 296,300 & .800 & .871 & -.071 \\
& & .779 & .255 & .524 \\
3 & 70,69 & $\mathrm{C}$ & & \\
& 69,70 & .594 & .712 & -.118 \\
6 & 300,296 & .608 & .736 & -.128 \\
& 296,300 & .586 & .689 & -.103 \\
& & .585 & .606 & -.021 \\
3 & 70,69 & $\mathrm{~L}$ & & \\
& 69,70 & .676 & .738 & -.062 \\
6 & 300,296 & .664 & .578 & .086 \\
& 296,300 & .696 & .816 & -.120 \\
& & .666 & .313 & .353 \\
3 & 70,69 & $\mathrm{R}$ & & \\
& 69,70 & .637 & .582 & .055 \\
6 & 300,296 & .659 & .798 & -.139 \\
& 296,300 & .693 & .338 & .355 \\
& & .721 & .798 & -.077 \\
\hline
\end{tabular}

Note--B\&P, Baranski and Petrusic (1994); C, stimulus combinations in middle; $L$, stimulus combination $3 \mathrm{~mm}$ to left of center; $R$, stimulus combination $3 \mathrm{~mm}$ to right of center.

experiment. Ten subjects spontaneously answered that they had used the monitor edges ( 3 in the $C$ condition, 4 in the $\mathrm{L}$ condition, and 3 in the $\mathrm{R}$ condition), 20 did so when explicitly asked ( 6 in the $\mathrm{C}$ condition, 7 in the $\mathrm{L}$ condition, and 7 in the $\mathrm{R}$ condition), and 12 subjects did not report having used the strategy ( 5 in the $C$ condition, 3 in the $\mathrm{L}$ condition, and 4 in the $\mathrm{R}$ condition). Subjects also postrated the total percentage of correct responses they had made in the experiment, and the ratings did not differ much from mean confidence ratings. The mean postratings (pr), mean confidence $(\bar{x})$, and proportion correct $(\bar{c})$ for the $\mathrm{C}, \mathrm{L}$, and $\mathrm{R}$ conditions were $\mathrm{pr}=.657(\bar{x}=.649$, $\bar{c}=.846) ; \mathrm{pr}=.739(\bar{x}=.720, \bar{c}=.780) ; \mathrm{pr}=.675$ $(\bar{x}=.701, \bar{c}=.757)$. Collapsed over all groups, $\mathrm{pr}=.690$ $(\bar{x}=.690, \bar{c}=.794)$. Subjects were obviously as underconfident in their postratings as in their confidence ratings during the experiment. Finally, there was a low correlation between postratings of proportion correct and actual proportion correct $[r(40)=.062, p>.69]$.

\section{Discussion}

The results of Experiment 1 were in accordance with all the hypotheses stated earlier; a lower proportion of correct responses but higher mean confidence ratings were observed in the $L$ and $R$ groups than in the $C$ group. Apparently, slightly moving the stimulus combinations either to the left or to the right caused subjects to become misled by the setting and highly reduced the underconfidence phenomenon, and this turned into overconfidence for hard stimuli. The differentiated pattern observed in the $L$ and $\mathrm{R}$ groups together with the verbal reports show that subjects did rely on the edge strategy. If there is a "left looks farther away" illusion, this illusion is not very impressive, for it can be reversed into a "right looks farther away" illusion by a small experimental manipulation. The almost identical positional-order effects found in the $L$ condition and in the data observed by $B \& P$ are suggestive. We cannot, of course, prove that B\&P actually used a particular experimental setting, but the only way in which we can replicate their pattern of results is to misalign our stimulus display to the left. Even if the whole screen was not off center in relation to the monitor edges, the same effect could be obtained with irregularities in the screen or per-

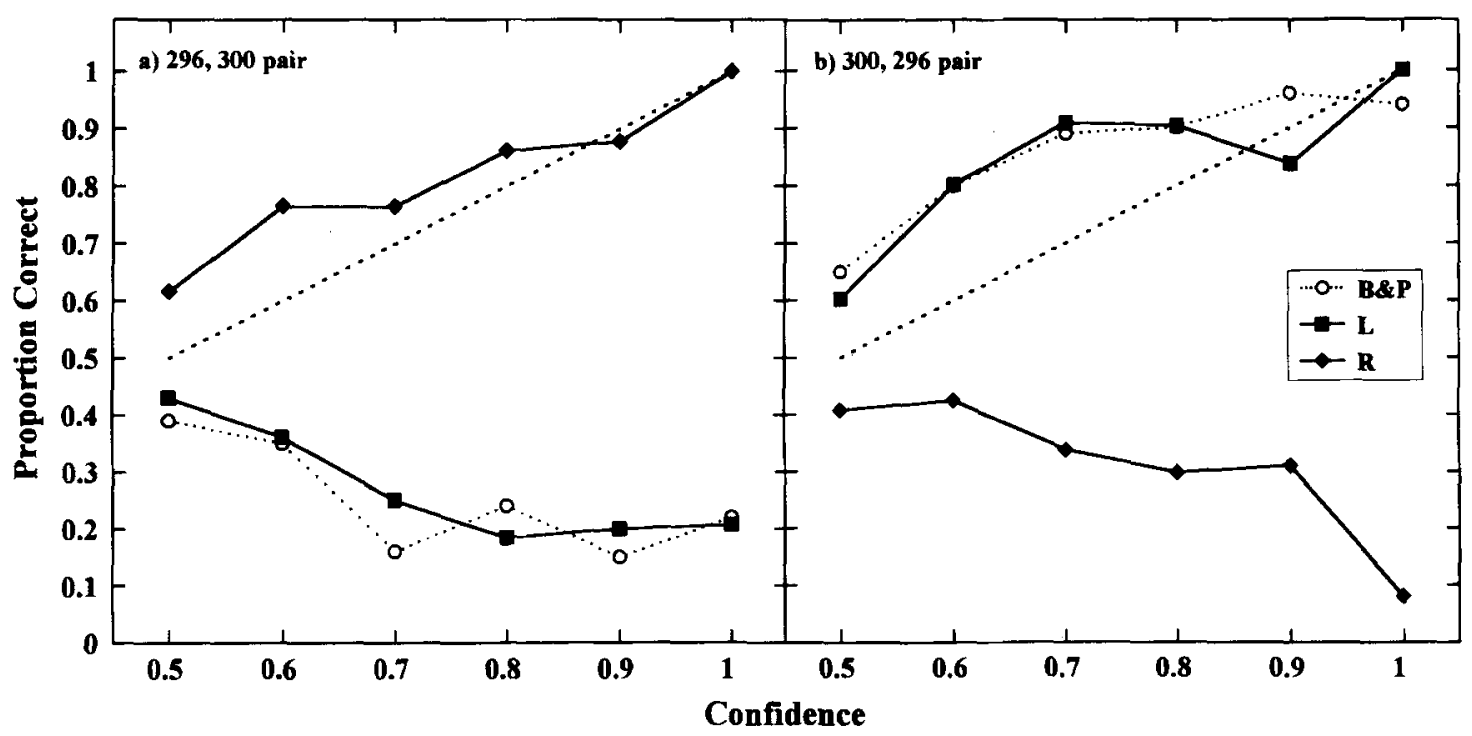

Figure 3. Experiment 1: calibration curves (a) for pair $(296,300)$, for the $L$ and $R$ conditions, and (b) for pair (300, 296), for the $L$ and $R$ conditions, compared with data estimated from Figure 7 in Baranski and Petrusic (1994). 
haps by a particular location and orientation of the subject's chair. However, we find our explanation of their results to be very likely correct. The overconfidence effects observed in both of our "distorted" experimental groups are of similar magnitude but are smaller than the effects found by B\&P. For example, in Pair 6, we observe $\mathrm{O} / \mathrm{U}$ values of .353 and .355 , whereas $B \& P$ reported .524 . This difference may be attributed to the arbitrariness in the distance chosen $(3 \mathrm{~mm})$ for the off-centering of the stimulus combinations. This can probably also account for the overall lower confidence ratings observed in both of our misaligned conditions.

B\&P have stated that "people can be overconfident on perceptual judgments if the level of the difficulty of the judgments is sufficiently high" (p. 417). Obviously, given a proportion correct of .25 and a scale that begins with $50 \%$ (remember that responses under $50 \%$ were counted as errors), the subjects do not even stand a chance of not being overconfident. However, a moderate overconfidence is also to be expected for almost indiscriminable stimuli with a proportion correct of about .50 , for the following reasons. First, subjects probably expect to use, at least in some instances, the entire range of the confidence scale. Being given a confidence scale ranging up to $100 \%$ might simply imply that one should use the higher categories. Second, subjects have some prior expectation of task difficulty, implying that they do not believe that they will be given an impossible task. Third, and perhaps most importantly, confidence ratings are subject to error fluctuations. As one approaches a proportion of correct responses of .5, these errors will all work in one direction and lead to a slight overconfidence. (See Erev, Wallsten, \& Budescu, 1994 , for a discussion of the role of error in judgment processes.) Thus, the overconfidence found in tasks in which stimuli are almost indiscriminable can be explained as a consequence of floor effects. A similar argument can be made when one comes close to $100 \%$ correct responses. Because of the ceiling effect, only underconfidence can be observed. However, it seems unlikely that this mechanism alone can account for the large underconfidence found for the easy subset of Experiment 1, in which the proportion of correct responses was .953 and the average confidence was .710 in the control condition (see Table 1).

To summarize: We found no support for B\&P's claim that overconfidence replaces underconfidence for proportions of correct responses below $80 \%$, and we have presented a plausible explanation for the results in B\&P's Experiment 2 , based on the interaction between experimental setting and response strategies.

\section{EXPERIMENT 2}

As noted above, less underconfidence is expected as we approach a proportion of correct responses of .5. In order to find out whether underconfidence can be obtained for very difficult items we designed Experiment 2, in which subjects were given an almost indiscriminable task with the screen edges removed (remember that it is easier to rely on the distance to the monitor side than on the dis- tance to the central line). Experiment 2 was also conducted to examine whether a positional-order effect could be obtained if the possibility of using the edge strategy was removed. It was expected that (1) the proportion of correct responses would be lower than in the control condition in Experiment 1 because the screen edges were removed, (2) no positional-order effect would be found, and (3) a slight overconfidence would be found for stimuli with a low proportion of correct responses (i.e., when the proportion of correct responses was close to .5). Experiment 2 was similar to Experiment 1, the main difference being that a box with a viewing hole was placed around the monitor, making the edges of the monitor invisible. Only two pairs were used: $(296,300 ; 300,296)$ and $(327$, $330 ; 330,327)$.

The reason for using the same pair $(296,300 ; 300,296)$ as in Experiment 1 was twofold: first, since it was hypothesized that subjects use the monitor edges, the proportion of correct responses would decrease if this opportunity was taken away from them. This would allow a further test of the hypothesis that subjects do rely on the edge strategy if possible. Second, if there is a genuine "left looks farther away" illusion that does not depend on the monitor edges, this illusion would show up clearly under the more controlled conditions of this experiment.

\section{Method}

Subjects. Twelve undergraduate students of Uppsala University ( 8 males and 4 females; mean age, 21.3 years) participated in the study. The subjects did not receive monetary reward but were given credit for a course requirement. All subjects had normal vision. The subjects were not informed of the purpose of the experiment, which lasted about $30 \mathrm{~min}$.

Apparatus. The same apparatus as that in Experiment 1 was used. In addition, a box with a viewing hole was placed around the computer monitor. The outer edges of the monitor were thus made invisible. Since subjects could not use the keyboard with this procedure, confidence responses were obtained by mouse operation on a scale shown directly on the computer screen. It was completely dark in the room during the experiment.

Stimuli. The stimulus display was the same as in Experiment 1. Two stimuli were used in the experiment, Pair $1(296,300 ; 300,296)$ and Pair $2(327,330 ; 330,327)$.

Procedure. The procedure was similar to that of Experiment 1. The difference was that instead of the visual prompt CONFIDENCE $\rightarrow$, a scale anchored with " $50 \%$ " and " $100 \%$ " appeared in the lower part of the screen. If subjects wanted to respond below $50 \%$, they used the mouse pointer to click on a button ("!") below the scale, and the scale then changed to " 0 " and " $49 \%$." The scale was a rectangle $(10 \times 200 \mathrm{~mm})$ with a vertical line that could be moved by the mouse pointer. The value that the line was set at was also shown below the scale. After setting the line, the subjects clicked on a button ("> "), also below the scale. This cleared the screen, and, as in Experiment 1, a 2-sec interval separated the trials. All combinations of the stimulus pairs were presented to each subject 30 times, making a total of 120 items. Each subject received six practice trials.

\section{Results}

The data were collapsed over FARTHER and NEARER trials, since no significant differences were found for proportion correct and confidence. The main results of Experiment 2 appear in Table 3. As predicted, Stimulus Pair $\mathbf{l}$ is associated with a lower proportion of correct re- 
Table 3

Experiment 2: Mean Confidence $(\bar{x})$, Proportion Correct $(\bar{c})$, and Over-/Underconfidence $(O / U$, i.e., $\bar{x}-\bar{c})$ for Pair 1 and Pair 2

\begin{tabular}{ccccc}
\hline Pair & Order, Pixels & $\bar{x}$ & $\bar{c}$ & $\mathrm{O} / \mathrm{U}(\bar{x}-\bar{c})$ \\
\hline 1 & 296,300 & .553 & .598 & -.045 \\
& 300,296 & .566 & .592 & -.026 \\
$M$ & & .560 & .595 & -.035 \\
2 & 327,330 & .550 & .555 & -.005 \\
& 330,327 & .557 & .559 & -.002 \\
$M$ & & .553 & .557 & -.004 \\
\hline
\end{tabular}

sponses in this experiment than was the same stimulus pair in the control condition of our Experiment $1[t(24)=$ $2.23, p<.05]$. For this stimulus pair, subjects are underconfident. Although the hardest stimulus pair was not quite as hard as anticipated $(\bar{c}=.555)$, it was still harder than the hard level of Experiment 2 in B\&P. It is interesting to note that even at such low levels of proportion of correct responses, subjects are not overconfident $(\mathrm{O} / \mathrm{U}=-.035$ and $\mathrm{O} / \mathrm{U}=-.004$ for the two pairs, respectively). Results pertinent to any positional-order effect can also be found in Table 3. It can be seen that the proportions of correct responses are essentially identical whether the left stimulus is farthest from or nearest to the central marker, with no. sign of a positional-order effect.

The relative frequency of responses in the different confidence categories were $.5(71.45 \%), .6(14.50 \%), .7$ $(4.25 \%), .8(5.65 \%), .9(4.11 \%)$, and $1.0(0 \%)$. It can be noted that $85 \%$ of the responses were located in the two lowest confidence categories and that no subjects used the $100 \%$ category.

To summarize: Experiment 2 showed that (1) when the possibility of using the monitor edges is removed, the proportion of correct responses is reduced; (2) there is nothing supporting a "left looks farther away" illusion under carefully controlled conditions; and (3) even at a proportion correct of .555, subjects show no sign of overconfidence.

\section{GENERAL DISCUSSION}

In this paper we have made two points, one concerned with the possibility of overconfidence in sensory discrimination and one methodological. In Experiment 1, it was shown how easily experimental setting and response strategies influence results. Specifically, we showed that when the stimulus combinations were properly centered, underconfidence was found for all levels of difficulty. This result is in clear contrast with B\&P's observations of good calibration for difficulty levels yielding approximately $80 \%$ correct responses, underconfidence above $80 \%$, and overconfidence below $80 \%$. Even in the most difficult condition of our Experiment 2, overconfidence was not found. Overall, the size of the underconfidence found in the control condition in Experiment 1 was large $(\mathrm{O} / \mathrm{U}=-.197)$ in comparison with other empirical data (see Björkman et al., 1993).

We have also presented a plausible explanation of the results of $B \& P$ 's Experiment 2 -namely, that subjects take the monitor edges into consideration when making their judgments. It was proposed that a slight off-centering of the stim- ulus combinations (i.e., what is presented on the screen is off center relative to the monitor edges) in a particular direction would lead to the kind of positional-order effect reported by B\&P. This was confirmed empirically. The effect of stimulus position found by B\&P was (1) reproduced, (2) eliminated, and (3) reversed under conditions predicted by the hypotheses put forward. Thus, full experimental control of the findings reported by $\mathrm{B} \& \mathrm{P}$ has been demonstrated.

This paper has been directed at Experiment 2 in B\&P's study, but the explanation put forward here could also hold for their Experiment 1, which was conducted in the same way, with the use of the same apparatus, as was Experiment 2 . The authors reported that the positional-order effect was not evident in their Experiment 1 , but that it could have been masked by the higher proportion of correct responses. The authors observed in their Experiment 1 an overall proportion of correct responses of .915 and an underconfidence of only -.054 . The reported accuracy level is close to our "medium" level, where we observe a proportion of correct responses of .900 and a large underconfidence of -.256 . Other phenomena reported by $\mathrm{B} \& \mathrm{P}$ can be accounted for by our hypotheses. The authors'observation of a hard-easy effect may in part be explained by the fact that for hard items subjects rely more on "the edge strategy" and accordingly are being more misled on those items. Winman and Juslin (1993) showed that realism of confidence improved by extensive ( 160 trials) trial by trial outcome feedback in a cognitive task (general knowledge items), but not in a sensory discrimination task (lines). The effect of feedback on resolution found by $B \& P$ could possibly be understood in terms of the partly cognitive strategy used by subjects.

One of the most important issues in the debate regarding the proposed phenomenon of underconfidence in sensory discrimination centers on the questions as to whether there is a difference between confidence in sensory and cognitive judgments and whether one model can explain the phenomenon 's pertaining in both domains (Björkman et al., 1993, 1995; Ferrell, 1994, 1995; Juslin \& Winman, 1995). $B \& P$ repeatedly stress that "there are a number of similarities between the properties of confidence calibration in perceptual and nonperceptual tasks" (p. 424). In contrast to this, we suggest that there are psychologically relevant differences between cognitive and sensory judgments.

First, the data in our paper suggest that in some sensory judgments there is no hard-easy effect in the same sense as in cognitive judgments (i.e., overconf idence below $80 \%$ correct and underconfidence over $80 \%$ correct). For the natural reasons discussed above, we observe a larger underconfidence for very high proportions correct than for very low, owing to response error and foor/ceiling effects. These regression effects probably do not reveal anything interesting about the mind of the human decision maker It seems to be much more difficult to obtain overconfidence in sensory judgments than in cognitive, especially in pair-comparison tasks where subjects can inspect the stimuli as long as they wish, or "subject-controlled" studies (see Vickers et al., 1985). Under these conditions, a pervasive underconfidence bias was observed in the pres- 
ent experiments and in Björkman et al. (1993). When these conditions are not met-for example, when stimuli are not continuously presented for inspection by the subjects, as in Experiment 3 in $\mathrm{B} \& \mathrm{P}$ - a lesser degree of underconfidence is observed.

Second, one important difference between confidence judgments in the sensory and cognitive domains occurs in how responses are correlated between subjects. Juslin et al. (1995) suggested that sensory discriminations are best modeled by a response-independence model. Such a model is appropriate when erroneous responses reflect random fluctuations in the nervous system that are independent across subjects (Thurstone, 1927). Cognitive judgments, on the other hand, are more appropriately modeled in terms of a response-dependence model, wherein responses are assumed to be correlated across subjects. Subjects may rely on the same probabilistic cue, which in turn can make them come up with the same answer for a particular item. An implication of these models, that solution probabilities of items show a greater range for cognitive than for sensory tasks, was shown by Juslin et al. (1995).

These observed differences suggest that one should not uncritically draw parallels between confidence in sensory and cognitive judgments. Of course, even if one were to find similar results for sensory and cognitive judgments, the possibility of quite different underlying processes could still not be ruled out. We believe that a deeper understanding of both similarities and differences between the processes operating in çognitive and sensory judgments is needed in order to achieve progress in the field. At this point, we must conclude that there is no satisfactory explanation of why underconfidence seems to be a pervasive finding in some sensory discriminations. The cognitive domain has certainly benefited from the last few years' development of theories (see, e.g., Björkman, 1994; Erev et al., 1994; Gigerenzer et al., 1991; Juslin, 1994). However, we cannot but agree with B\&P that the existing attempts to model confidence in sensory discrimination are not adequate. A theory explaining confidence in sensory discrimination is needed.

\section{REFERENCES}

Baranski, J. V., \& Petrusic, W. M. (1994). The calibration and resolution of confidence in perceptual judgments. Perception \& Psychophysics, 55, 412-428.

BJöRKMAN, M. (1994). Internal cue theory: Calibration and resolution of confidence in general knowledge. Organizational Behavior \& Human Decision Processes, 58, 386-405.

Buörkman, M., Juslin, P., \& Winman A. (1993). Realism of confidence in sensory discrimination: The underconfidence phenomenon. Perception \& Psychophysics, 54, 75-81.

Björkman, M., Juslin, P., \& Winman A. (1995). Reply to William R. Ferrell's paper "A model for realism of confidence judgments: Implications for underconfidence in sensory discrimination." Perception \& Psychophysics, 57, 255-259.

DAWES, R. M. (1980). Confidence in intellectual judgments vs. confidence in perceptual judgments. In E. D. Lanterman \& H. Feger (Eds.), Similarity and choice: Paper in honor of Clyde Coombs (pp. 327-345). Bern: Hans Huber.

Erev, I., Wallsten, T. S., \& Budescu, D. V. (1994). Simultaneous overand underconfidence: The role of error in judgment processes. Psychological Review, 3, 519-527.
FERRELL, W. R. (1994). Calibration of sensory and cognitive judgments: A single model for both. Scandinavian. Journal of Psychology, 35, 297-314.

FERRELL, W. R. (1995). A model for realism of confidence judgments: Implications for underconfidence in sensory discrimination. Perception \& Psychophysics, 57, 246-254.

Gigerenzer, G., Hoffrage, U., \& Kleinbölting, H. (1991). Probabilistic mental models: A Brunswikian theory of confidence. Psychological Review, 98, 506-528.

JuSLIN, P. (1993a). An ecological model of realism of confidence in one's general knowledge (Acta Universitatis Upsaliensis: Studia Psychologica Upsaliensia 14). Stockholm: Almqvist \& Wiksell.

JusLIN, P. (1993b). An explanation of the hard-easy effect in studies of realism of confidence in one's general knowledge. European Journal of Cognitive Psychology, 5, 55-71.

JusLIN, P. (1994). The overconfidence phenomenon as a consequence of informal experimenter-guided selection of almanac items. Organizational Behavior \& Human Decision Processes, 57, 226-246.

JusLIN, P. (1995). Well calibrated confidence judgments for general knowledge items, inferential recognition decisions and social predictions. In J.-P. Caverni, M. Bar-Hillel, F. H. Barron, \& H. Jungerman (Eds.), Contributions to decision making I (pp. 233-253). Amsterdam: Elsevier.

Juslin, P., \& Winman, A. (1995). Reply to William R. Ferrell's paper "Calibration of sensory and cognitive judgments: A single model for both." Scandinavian Journal of Psychology, 36, 153-163.

Juslin, P., Winman, A., \& Persson, T. (1995). Can overconfidence be used as an indicator of reconstructive rather than retrieval processes? Cognition, 54, 99-130.

KEREN, G. (1988). On the ability of monitoring non-veridical perceptions and uncertain knowledge: Some calibration studies. Acta Psychologica, 67, 95-119.

Keren, G. (1991). Calibration and probability judgments: Conceptual and methodological issues. Acta Psychologica, 77, 217-273.

Lichtenstein, S., Fischhoff, B., \& Phillips, L. D. (1982). Calibration of subjective probabilities: The state of the art up to 1980. In D. Kahneman, P. Slovic, \& A. Tversky (Eds.), Judgments under uncertainty: Heuristics and biases (pp. 306-334). New York: Cambridge University Press.

O'CoNNOR, M. (1989). Models of human behaviour and confidence in judgment: A review. International Journal of Forecasting, 5, 159-169.

Thurstone, L. L. (1927). A law of comparative judgment. Psychological Review, 34, 273-286.

Vickers, D., Smith, P., Burt, J., \& Brown, M. (1985). Experimental paradigms emphasizing state or process limitations: II. Effects on confidence. Acta Psychologica, 59, 163-193.

WALlSTEN, T. S., \& BUDESCU, D. V. (1983). Encoding subjective probabilities: A psychological and psychometric review. Management $S_{C i}$ ence, 29, 152-173.

WINMAN, A. (in press). The importance of item selection in "knew-itall-along" studies of general knowledge. Scandinavian Journal of Psychology.

Winman, A., \& JusLin, P. (1993). Calibration of sensory and cognitive judgments: Two different accounts. Scandinavian Journal of Psychology, 34, 135-148.

YATES, J. F. (1990). Judgment and decision making. Englewood Cliffs, NJ: Prentice-Hall.

\section{NOTE}

1. B\&P's way of presenting data in their Figures 1,4 , and 7 deviates somewhat from common procedures. Given that all confidence assessments in, for example, the category .50 lie in the range $.50-.59$, mean confidence actually lies above .50 . It is more common to calculate the actual mean confidence for each interval and let this value mark the category on the confidence scale. For the sake of making direct comparisons easy, however, we present the data in the same way as do B\&P. In the experiments reported here, the difference between the two ways of looking at data is small, because most subjects spontaneously make judgments in the exact intervals $.5, .6, .7, .8, .9$, and 1.0 .

(Manuscript received December 7, 1994; revision accepted for publication August 2, 1995.) 